\title{
Phonons and magnons in stripe-ordered nickelates. A Raman scattering study
}

\author{
V. Gnezdilov ${ }^{1}$, V. Kurnosov¹, Yu. Pashkevich² ${ }^{2}$ P. Lemmens ${ }^{3}$, J. Tranquada4 ${ }^{4}$ \\ K.-Y. Choi ${ }^{5}$, G. Güntherodt ${ }^{5}$, K. Nakajima ${ }^{6}$, and A. Yeremenko ${ }^{1}$ \\ ${ }^{1}$ B. Verkin Institute for Low Temperature Physics and Engineering of the National Academy of \\ Sciences of Ukraine, 47 Lenin Ave., Kharkov 61103, Ukraine \\ E-mail: kurnosov@ilt.kharkov.ua \\ ${ }^{2}$ A. Galkin Donetsk Physico-Technical Institute of the National Academy of Sciences of Ukraine \\ 72 R. Luxemburg Str., Donetsk 83114 , Ukraine \\ ${ }^{3}$ MPI for Solid State Research, MPI-FKF, Stuttgart D-70569, Germany \\ ${ }^{4}$ Brookhaven National Laboratory, Upton, New York 11973, USA \\ ${ }^{5}$ Physikalisches Institut, RWTH Aachen, Aachen 52056, Germany \\ ${ }^{6}$ Neutron Scattering Laboratory ISSP, University of Tokyo, Tokai, Ibaraki, Japan
}

Received June 8, 2004, revised September 2, 2004

\begin{abstract}
Electronic correlation effects in $\mathrm{La}_{2-} \mathrm{Sr}_{x} \mathrm{NiO}_{4}(x=1 / 3$ and 0.225$)$ lead to spontaneous phase separation into microscopic spin/charge stripes with commensurate and incommensurate order, respectively. Raman scattering experiments on such single crystalline materials show a rich phenomenology of phonon and magnon anomalies due to the new, self-organized periodicities. These effects are observable as function of temperature but can also be induced by cooling in a seemingly small magnetic field leading to a reorganization of stripe structure.
\end{abstract}

PACS: 72.10.Di, 71.27.+a, 78.30.-j

\section{Introduction}

Stripe ordering of charge and spin in transition-metal oxides has been of intense interest in condensed-matter physics from the theoretical and experimental point of view as an example of a nontrivial ordering phenomenon that originates from the interplay between charge hybridization and interaction. Historically, the first evidence for unusual magnetic correlations was obtained in doped nickel oxide, namely on a single crystal of $\mathrm{La}_{1.8} \mathrm{Sr}_{0.2} \mathrm{NiO}_{3.96}$ in a neutron diffraction study [1]; similar magnetic ordering was also observed in $\mathrm{La}_{2} \mathrm{NiO}_{4.125}$ [2]. A second set of superlattice peaks, indicative of charge order, was detected in a series of $\mathrm{La}_{2}{ }_{-x} \mathrm{Sr}_{x} \mathrm{NiO}_{4+\delta}$ samples by electron diffraction [3]. Neutron diffraction studies [4,5] on a crystal of $\mathrm{La}_{2} \mathrm{NiO}_{4+\delta}$ with $\delta=0.125$ were the first to detect the magnetic and charge-order superstructure peaks simultaneously. The observed su- perstructure provided clear evidence for a highly correlated state in which the dopant-induced holes segregate into periodically spaced stripes that separate antiferromagnetic domains. Later a static form of this modulation has been clearly identified in the system $\mathrm{La}_{1.6-x} \mathrm{Nd}_{0.4} \mathrm{Sr}_{x} \mathrm{CuO}_{4}$ [6], where an anomalous suppression of superconductivity was found for $x$ $\sim 1 / 8$. A model of a dynamical form of this modulation was exploited for interpreting the spin correlations in $\mathrm{La}_{2-x} \mathrm{Sr}_{x} \mathrm{CuO}_{4}$ [7] with $x \neq 1 / 8$ and $\mathrm{YBa}_{2} \mathrm{Cu}_{3} \mathrm{O}_{6+\delta}$ [8].

Here we will focus mainly on the $\mathrm{La}_{2-x} \mathrm{Sr}_{x} \mathrm{NiO}_{4+\delta}$ system, which exhibits stripe ordering over wide range of hole concentration [3,4]. Before continuing, it may be useful to review briefly some of the basic knowledge, notations, and relevant work. The structure of the parent compound $\mathrm{La}_{2} \mathrm{NiO}_{4}$ consists of $\mathrm{NiO}_{2}$ planes separated by $\mathrm{La}_{2} \mathrm{O}_{2}$ layers. Within a $\mathrm{NiO}_{2}$ plane, $\mathrm{Ni}$ ions form a square lattice with oxygen atoms bridging 
the nearest-neighbor sites. The unit-cell vectors $\mathbf{a}_{1}$ and $\mathbf{a}_{2}$ are parallel to nearest-neighbor $\mathrm{Ni}-\mathrm{O}$ bonds within the planes, and $\mathbf{a}_{3}$ is perpendicular to the planes. There are two $\mathrm{NiO}_{2}$ planes per unit cell, and they are related by the basic vector $1 / 2 \mathbf{a}_{1}+1 / 2 \mathbf{a}_{2}+1 / 2 \mathbf{a}_{3}$. For each $\mathrm{Ni}$ ion there is one out-of-plane oxygen atom directly above and one atom below (along $\mathbf{a}_{3}$ axis) effectively completing a tetragonally-distorted octahedron of oxygen ions. La ions sit above and below the centers of the squares formed by the $\mathrm{Ni}$ ions. The simple structure described above is known as the High Temperature Tetragonal (HTT) phase of the $\mathrm{K}_{2} \mathrm{NiF}_{4}$ structure. Upon cooling, $\mathrm{La}_{2} \mathrm{NiO}_{4}$ undergoes two structural phase transitions at 650 and $75 \mathrm{~K}$. Thus, with decreasing temperature, the phase transitions (and space groups) are

\section{HTT $(\mathrm{I} / / \mathrm{mmm}) \rightarrow$ LTO $(\mathrm{Abma}) \rightarrow$ LTT $\left(\mathrm{P}_{2} / \mathrm{ncm}\right)$.}

The $\mathrm{NiO}_{2}$ planes can be doped with holes both by $\mathrm{Sr}$ substitution and by addition of excess oxygen. However, contrary to conventional expectations, the material remains nonmetallic up to quite large hole concentrations [9-11]. The insulating behavior occurs because the dopant-induced holes tend to order themselves in periodically spaced stripes. Nevertheless, these is considerable evidence for one-dimensional charge transport along the charge rows in the static stripe ordered phase both for $\mathrm{La}_{2-x} \mathrm{Sr}_{x} \mathrm{NiO}_{4+\delta}$ and $\mathrm{La}_{2-x-y} \mathrm{Nd}_{y} \mathrm{Sr}_{x} \mathrm{CuO}_{4}$ systems [12,13]. These charge stripes run diagonally relative to the square lattice defined by the $\mathrm{Ni}-\mathrm{O}-\mathrm{Ni}$ bonds. In the essentially undoped regions between the stripes the $\mathrm{Ni}$ spins can order antiferromagnetically, with the charge stripes acting an antiphase domain walls $[1,2,4,5]$. The analysis of results on stripe order for a number of doped $\mathrm{La}_{2} \mathrm{NiO}_{4}$ shows that the charge orders at a higher temperature $\left(T_{c o}\right)$ than the spins $\left(T_{m}\right)$ and that both the $T_{c o}$ and $T_{m}$ increase systematically with holes concentration increasing [14]. This fact indicates the primary role of charge in driving the ordering.

The average structure of the compositions under study remains in the high-temperature tetragonal (HTT) phase (space group $14 / \mathrm{mmm}$ ) down to at least $10 \mathrm{~K}$ [15]. The charge and spin order are more easily described in a unit cell size $\sqrt{2} a \times \sqrt{2} a \times c$. Then, the charge density modulation is characterized by the wave vector $\mathbf{g}_{2 \varepsilon}=(2 \varepsilon, 0,0$,$) and the characteristic$ wave vector for the spin-density modulation is $\mathbf{g}_{\varepsilon}=$ $=(1+\varepsilon, 0,0$,$) (in real space modulation periods are$ $a / 2 \varepsilon$ and $a / \varepsilon$, respectively). In the first studies of $\mathrm{La}_{2-x} \mathrm{Sr}_{x} \mathrm{NiO}_{4}$ it has been suggested that ordering of the dopant-induced holes occurs only commensurately at special values of $x$, such as $1 / 2$ and $1 / 3[3,16]$. Later it was found that a single crystal with $x=0.2$, $a$

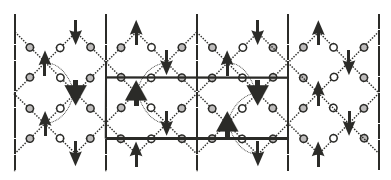

$b$

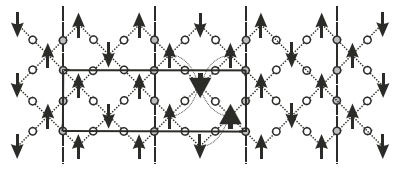

Fig. 1. Ni-centered domain walls (a); O-centered domain walls (b). Stripe models for $1 / 3$ doping [18]. Arrows indicate correlated Ni magnetic moments; circles indicate oxygen sites; filled circles indicate locations of doped holes on oxygen sites. Bold dashed lines indicate positions of domain walls, while bold solid lines outline a magnetic unit cell. The two-magnon Raman process is shown also: bold arrows demonstrate spins on adjacent sites and curved lines indicate broken magnetic bonds.

although not at a special value of $x$, shows commensurate order [17], albeit with a short in-plane correlation length of $\sim 40 \AA$. In contrast, the stripe order in $\mathrm{La}_{2} \mathrm{NiO}_{4+\delta}[4,5]$ and $\mathrm{La}_{1.775} \mathrm{Sr}_{0.225} \mathrm{NiO}_{4}$ [15] was found to be incommensurate, with the wave vector varying significantly with temperature. Since the stripes are charged, they will repel each other. As a result, the stripes will arrange themselves so as to maintain the maximum possible spacing, with the constrain that each stripe is centered on a $\mathrm{Ni}$ (site-centered stripes) or $\mathrm{O}$ (bond-centered stripes) site. For the case of $x=1 / 3$, it was shown [18] that in the temperature range $T_{c o}>T>T_{m}$ the domain walls are bond centered. For $T<T_{m}$ the density of stripes decreases, and the stripes become increasingly site centered. Stripe models for $x=1 / 3$ are illustrated in Fig. 1. In the real case, the spins are collinear and are shifted by angle $\varphi$ relatively the stripe direction $[19,20]$. It was found $\varphi=53^{\circ}$ at $T=14 \mathrm{~K}$ in $\mathrm{La}_{5} /{ }_{3} \mathrm{Sr}_{1 / 3} \mathrm{NiO}_{4}$ [21]. For the incommensurate stripe order, direct evidence for alternating site- and body-centered stripes within the $\mathrm{NiO}_{2}$ plane was presented in the transmission-electron-microscopy study of $\mathrm{La}_{1.725} \mathrm{Sr}_{0.275} \mathrm{NiO}_{4}$ crystal [22].

Despite very intense studies in the stripe physics field, it is somewhat surprising that there are only a few Raman scattering (RS) studies of this exotic form of order [12,23-26] and some deficiencies in our knowledge of light scattering in striped phases are now evident. For example, under the discussion is the question of RS from spin waves. Another problem, which has not been studied yet, is phonon dynamics in the direction perpendicular to the $\mathrm{NiO}_{2}$-planes.

\section{Experiment}

In our RS experiments two $\mathrm{La}_{2}-x \mathrm{Sr}_{x} \mathrm{NiO}_{4}(x=1 / 3$ and 0.225$)$ samples were studied. Single-crystals were grown by rf induction melting [27]. Measurements 
were performed in a backscattering configuration using Raman spectrometer DILOR XY with $5145 \AA$ laser light of $20 \mathrm{~mW}$. The incident laser beam was focused onto $0.1 \mathrm{~mm}$ diameter spot on the mirror-like polished and chemically cleaned crystal surface. The spectra were recorded on a liquid nitrogen-cooled $\mathrm{CCD}$. The laboratory coordinate system was locked to the axes of the crystal ( $x\|a, y\| b, z \| c$ ). The $x^{\prime}$ and $y^{\prime}$ axes are rotated by $45^{\circ}$ from $x$ and $y$. The $a, b$, and $c$ crystallographic axes in the $I 4 / \mathrm{mmm}$ setting were determined by $\mathrm{x}$ ray Laue diffraction. The measurements were performed in an optical cryostat in helium gas atmosphere. For the measurements in a magnetic field, the crystal was mounted in a cryostat with a horizontal-field superconducting magnet. The [110] axis of the crystal was aligned parallel to the magnetic field.

\section{Results and discussion}

For the tetragonal $\mathrm{K}_{2} \mathrm{NiF}_{4}$ structure, of the total twelve zone center phonon modes, four $\left(2 A_{1 g}+2 E_{g}\right)$ ones are Raman active. Figures 2 and 3 show room temperature RS spectra in $x^{\prime} x^{\prime}$ scattering geometry for both samples under study. In this geometry $A_{1}$ lines are allowed. First of them at around $230 \mathrm{~cm}^{-1}$ was assigned to the La stretching mode [28-31]. The second one at around $450 \mathrm{~cm}^{-1}$ was identified as the oxygen stretching mode [28-31]. Above the charge ordering

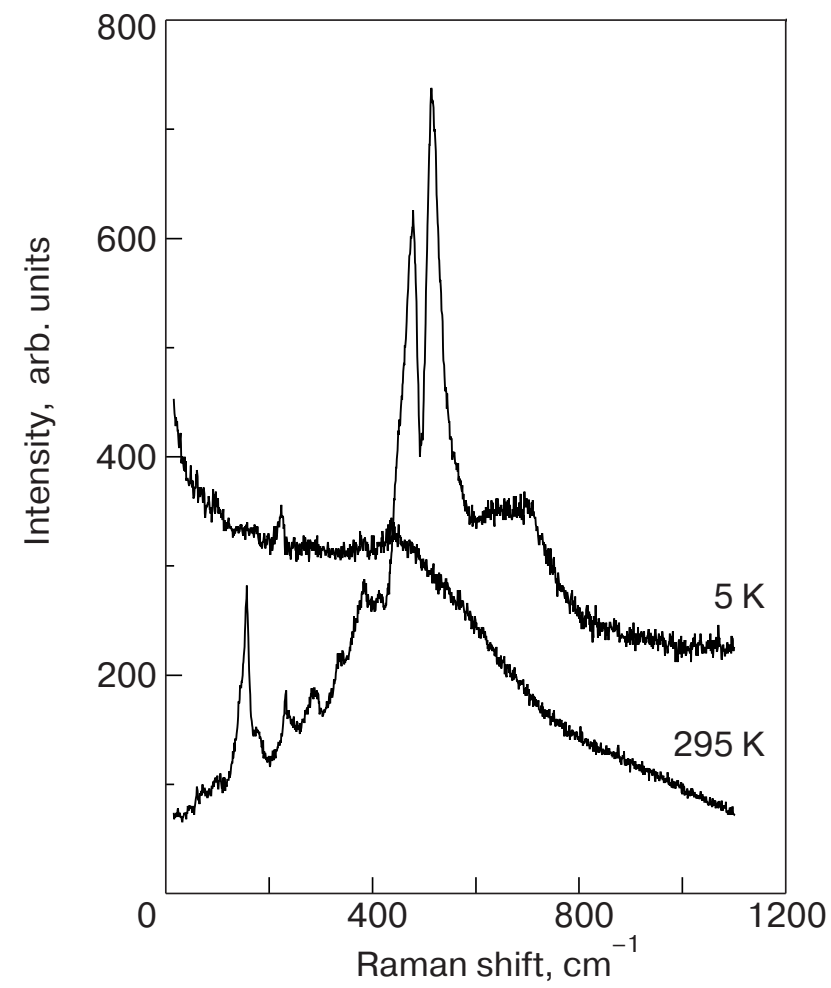

Fig. 2. The $x^{\prime} x^{\prime}$ Raman spectra of the single crystal $\mathrm{La}_{5} / \mathrm{Sr}_{1 / 3} \mathrm{NiO}_{4}$ at 5 and $295 \mathrm{~K}$.

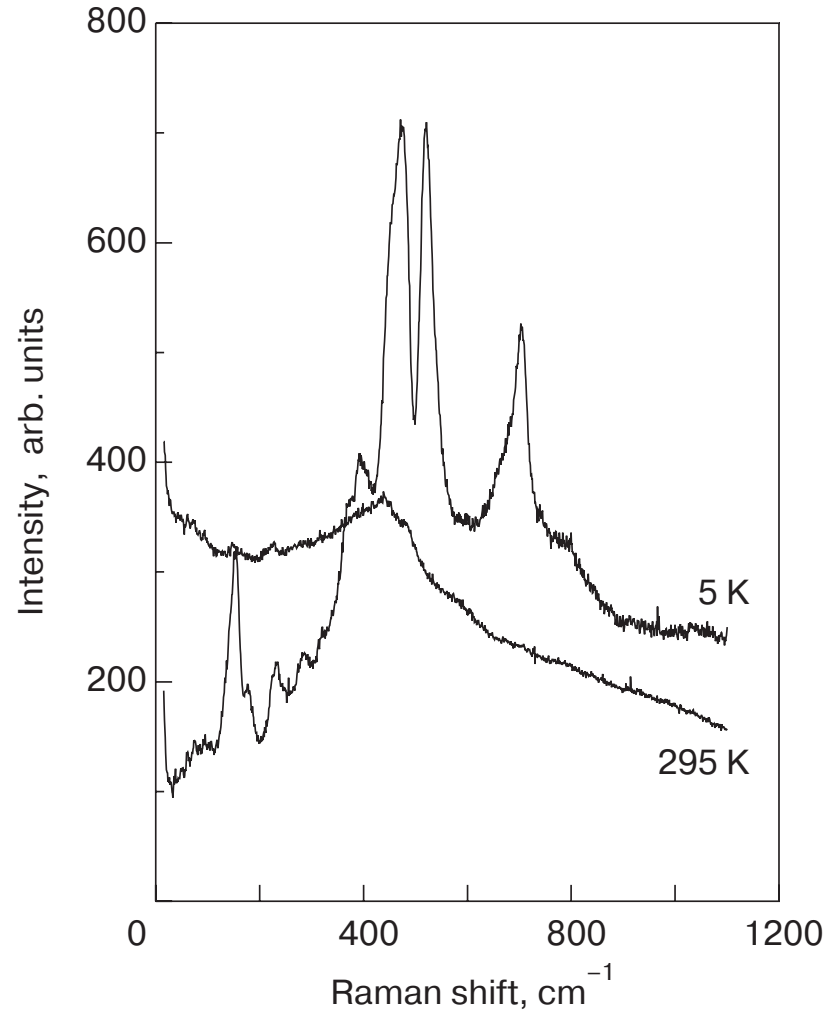

Fig. 3. The $x^{\prime} x^{\prime}$ Raman spectra of the single crystal $\mathrm{La}_{1.775} \mathrm{Sr}_{0.225} \mathrm{NiO}_{4}$ at 5 and $295 \mathrm{~K}$.

temperature all the observed modes are weak; the 230 and especially $450 \mathrm{~cm}^{-1}$ modes are broad, indicating strong polaronic effects and inhomogeneous charge distribution [23,32]. Notable changes in the RS spectra are observed below $T_{c o}$. The charge ordering gives rise to formation of a superlattice, multiplies the unit cell size, and lowers the crystal symmetry. It leads to the appearance of new $\Gamma$-point Raman-active phonon modes in the spectra. The origin of the extra lines in the stripe-ordered state and their assignment were made in Ref. 26.

Now we turn to the measurements in $z z$ polarization configuration. For the crystal with $x=1 / 3$ two lines of $A_{1 \mathrm{~g}}$ symmetry at 232 and $448 \mathrm{~cm}^{-1}$ are observed at room temperature as shown in Fig. 4. The disadvantage of the Sr-doped $\mathrm{La}_{2} \mathrm{NiO}_{4}$ system is that the dopant positions are fixed at relatively high temperature and may be random. At room temperature we do not see any dopant-induced extra features in the low frequency part of the spectra. It is possible also to assume a regular order of the Sr ions within the crystal structure in the special cases of doping $(x=1 / 2,1 / 3$, or $1 / 4)$ like the interstitial order in the oxygen-doped $\mathrm{La}_{2} \mathrm{NiO}_{4}$. The line shape of the $\mathrm{Ni}-\mathrm{O}_{2}$ bond stretching mode at $448 \mathrm{~cm}^{-1}$ is asymmetric. This asymmetry can be explained by a random distribution of holes on oxygen above $T_{c o}$. 


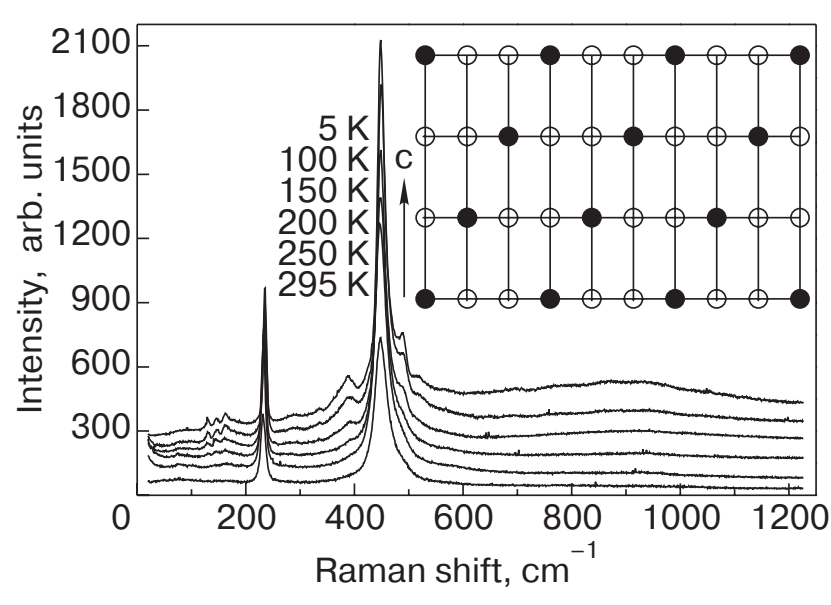

Fig. 4. Temperature dependent Raman spectra for $z z$ polarization of single crystal $\mathrm{La}_{5}{ }_{3} \mathrm{Sr}_{1}{ }_{3} \mathrm{NiO}_{4}$. Inset shows an idealized structure of the stripe-ordered phase in the plane perpendicular to the charge domain walls for $1 / 3$ doping. The open circles indicate correlated in the $\mathrm{NiO}_{2}$ layers spins at $\mathrm{Ni}^{2+}$ sites. The filled circles show locations of doped holes on $\mathrm{Ni}$ sites.

The changes in phonon spectra are observed below $T_{c o}-$ new phonon peaks at approximately 130, 145, $160,285,330,386,488$, and $520 \mathrm{~cm}^{-1}$ appear. To explain this, we ought to analyze the stripes alignment in the neighboring $\mathrm{NiO}_{2}$ layers. As it was supposed in earlier publications [5], the charge stripes align themselves from one layer to the next so as to minimize the long range part of the Coulomb interaction. However the pinning of the charge stripes to the lattice means that the shift of the stripe pattern from one layer to the next can only occur in increments of the lattice spacing. For this sample, with the stripe spacing of $3 / 2 a$ it is possible to have a perfectly body-centered stacking. Such a symmetric stacking of the layers of stripes can lead to forbidden superlattice peaks corresponding to the charge order. Inset on Fig. 4 shows the possible arrangement of the charge stripes in the neighboring layers for the $x=1 / 3$ crystal. In this case an additional periodicity along the $c$ axis can also lead to forbidden superlattice peaks that are most likely observed in our experiments.

Let us analyze now the situation with the $x=0.225$ composition (see Fig. 5). Whereas $x^{\prime} x^{\prime}$ and $x^{\prime} y^{\prime}$ spectra are very similar in both compounds, pronounced differences are observed in $z z$ scattering geometry. In contrast to the $x=1 / 3$ sample, the spectra of the $x=0.225$ sample even at room temperature have very complicated shape. Under lowering temperature, the shape does not change, with the exception of a continuum, which low-frequency portion reduces in intensity with temperature reduction. To explain this, we oblige to suppose that for the not special case (as $x=$

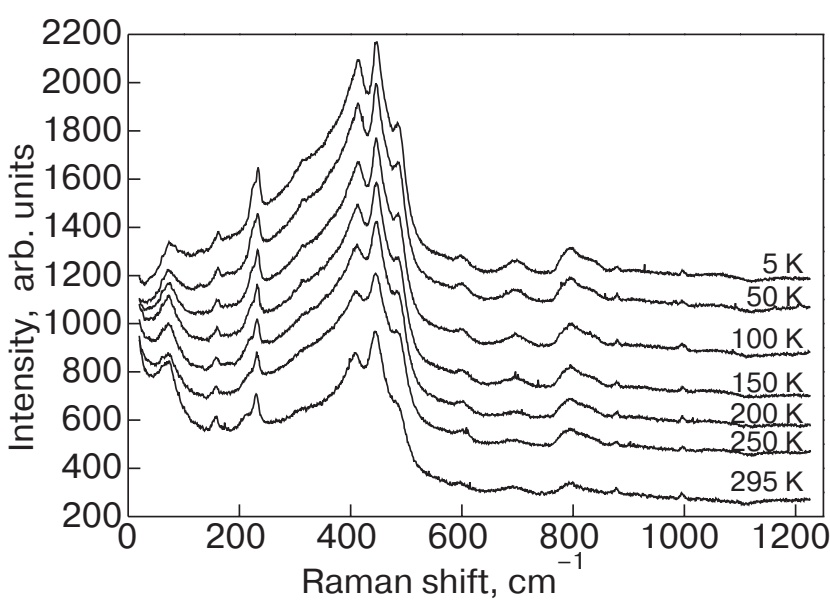

Fig. 5. Temperature dependent Raman spectra for $z z$ polarization in single crystal of $\mathrm{La}_{1.775} \mathrm{Sr}_{0.225} \mathrm{NiO}_{4}$.

$=1 / 2,1 / 3$, or $1 / 4$ ) the random dopant ions (and holes) distribution lead to a break of long ranger order in $c$ direction. In this case $\mathbf{k}$ conservation is not required and the first order Raman spectrum is a measure of the density of vibrational states. If this assumption is correct, stripe ordering below $T_{c o}$ should not result in the occurrence of new features in the spectra. Moreover, for our sample with an average stripe spacing of about $1.82 a$, it is not possible to have a perfectly body-centered stacking. A similar conclusion was firstly reached in neutron diffraction study [33] of the sample with average stripe spacing of about $1.75 a(x=0.275)$ and confirmed in the high-resolution transmission-electron-microscopy study [22].

For both $\mathrm{La}_{5}{ }_{3} \mathrm{Sr}_{1 / 3} \mathrm{NiO}_{4}$ and $\mathrm{La}_{1.775} \mathrm{Sr}_{0.225} \mathrm{NiO}_{4}$ two relatively strong bands at $\sim 720 \mathrm{~cm}^{-1}\left(740 \mathrm{~cm}^{-1}\right)$ and $\sim 1110 \mathrm{~cm}^{-1}\left(1130 \mathrm{~cm}^{-1}\right)$ were observed at low temperature in $x^{\prime} y^{\prime}$ polarization $[23,24,26]$. These bands were interpreted as two-magnon scattering $[23,24,26]$. What was the reason for this attribution? Two-magnon scattering involves a simultaneous excitation of a pair of magnons with equal and opposite momenta $\mathbf{k}$ on each of the sublattices. In total, excitations from the entire Brillouin zone lead to a band of Raman frequencies that reflects the magnon density of states. Since the density is sharply peaked at the zone-boundary, Raman scattering probes preferentially localize antiferromagnetic order. If two spin deviations are created on sites far apart, the excitation frequency is $2\left(\mathrm{JSz}+g \mu_{B} B_{A}\right)$, where $z$ is the number of nearest neighbors, $B_{A}$ is the effective anisotropy field, and $J$ is the exchange interacting constant. In the case of two spin deviations are created on adjacent sites, the excitation frequency is only $J(2 S z-1)+2 g \mu_{B} B_{A}$ because the presence of the first spin deviation leads to a reduction in the energy required for the second spin deviation. The undoped $\mathrm{La}_{2} \mathrm{NiO}_{4}$ antiferromagnetic 


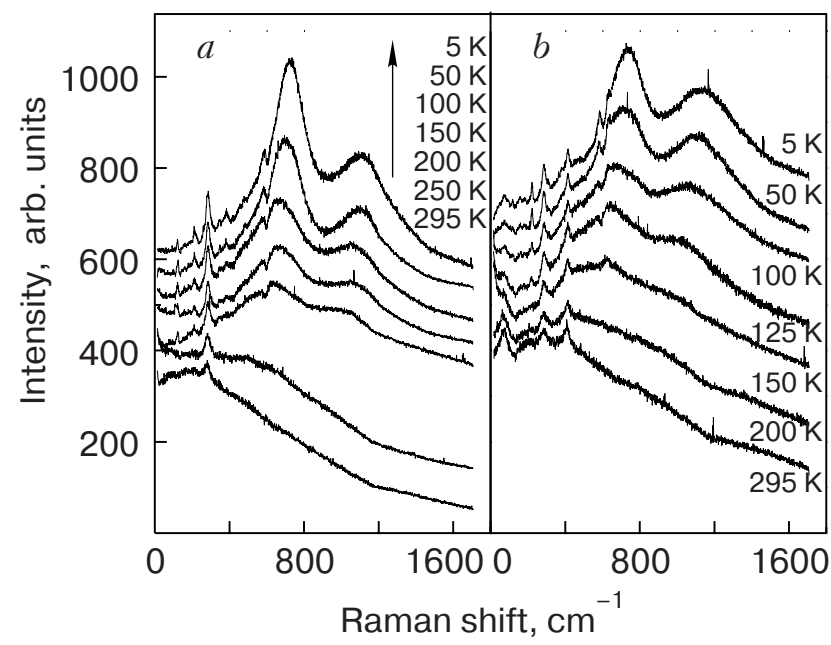

Fig. 6. Temperature dependent Raman spectra for $x^{\prime} y^{\prime}$ polarization of $\mathrm{La}_{5 / 3} \mathrm{Sr}_{1 / 3} \mathrm{NiO}_{4}(a)$ and $\mathrm{La}_{1.775} \mathrm{Sr}_{0.225} \mathrm{NiO}_{4}$ (b) single crystals.

insulator was studied by Sugai et al. [34]. The $B_{1 g}$ spectra exhibits a band peaked at $\sim 1640 \mathrm{~cm}^{-1}$ that has been assigned to a scattering by two-magnons. The estimated $J$ was $240 \mathrm{~cm}^{-1}$ on the assumption that the peak energy is $6.7 \mathrm{~J}$ for the $S=1$ nickel oxide.

For doped $\mathrm{La}_{2}-x \mathrm{Sr}_{x} \mathrm{NiO}_{4}$ the band near $1640 \mathrm{~cm}^{-1}$ was not observed in Raman experiments at any temperatures. Instead, two broad peaks in $x^{\prime} y^{\prime}$ polarization appear in the high frequency region under temperature lowering. Temperature dependent Raman spectra for $x^{\prime} y^{\prime}$ polarization of single crystals $\mathrm{La}_{2-x} \mathrm{Sr}_{x} \mathrm{NiO}_{4}(x=1 / 3$ and 0.225$)$ are shown in Fig. 6. In Fig. 7 the temperature dependence of the integrated intensity for both bands in the $x=0.225$ crystal is given. The first band was assigned to the two-magnon excitation within the antiferromagnetic

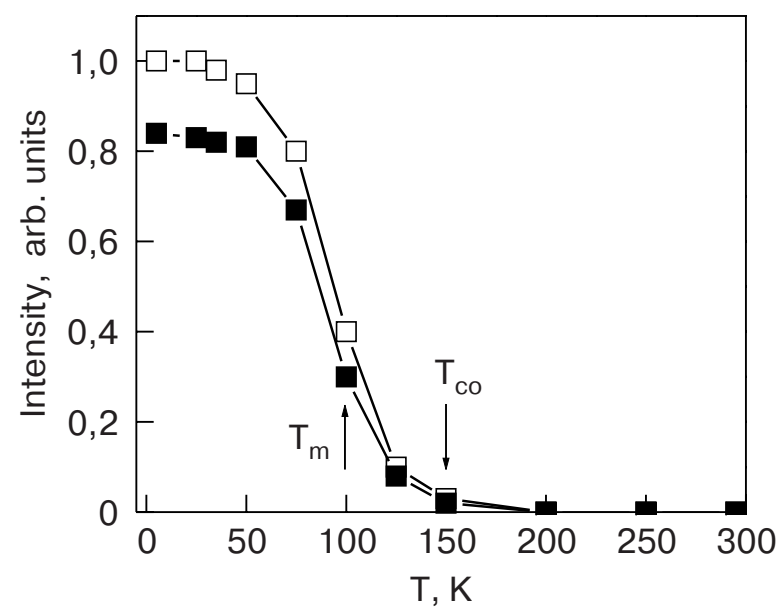

Fig. 7. The temperature dependence of the scattering intensity integrated above the charge carriers background for 740 ( $\square)$ and 1130 (ם) $\mathrm{cm}^{-1}$ bands in $\mathrm{La}_{1.775} \mathrm{Sr}_{0.225} \mathrm{NiO}_{4}$. domain and the second one to the excitation across the domain wall $[23,26]$ or to the excitation on the $\mathrm{Ni}^{2+}-\mathrm{Ni}^{3+}$ bond [24]. Accurate account of the spin-1 system gives peak positions of $\omega_{1} \approx 3 J$ and $\omega_{2} \approx 4 J$ for the $x=1 / 3 \mathrm{Ni}$-centered stripes. Thus, the peak positions for the two-magnon excitations within the antiferromagnetic domain and across the domain wall are $\omega_{1}=720 \mathrm{~cm}^{-1}$ and $\omega_{2}=960 \mathrm{~cm}^{-1}$ for the value of $J=240 \mathrm{~cm}^{-1}$, respectively. On the first sight it seems consistent with experiment.

Not recently this assignment was criticized in Ref. 35 . It was disclosed that the band at $\sim 730 \mathrm{~cm}^{-1}$ is due to a one-phonon excitation and only the $\sim 1120 \mathrm{~cm}^{-1}$ excitation is due to the magnetic excitation related to the stripe structure. The $685 \mathrm{~cm}^{-1}$ band, which appears in $\mathrm{La}_{2} \mathrm{NiO}_{4.15}$ at temperature lowering, was attributed to phonon excitation [25] because its energy coincides with one-phonon peak in $\mathrm{La}_{2} \mathrm{NiO}_{4.0}$. There are some doubts in last motivation by the following reasons.

1. The bands at $\sim 730 \mathrm{~cm}^{-1}$ in $\mathrm{La}_{2}{ }_{x} \mathrm{Sr}_{x} \mathrm{NiO}_{4}$ and at $685 \mathrm{~cm}^{-1}$ in $\mathrm{La}_{2} \mathrm{NiO}_{4.15}$ are much broader than one-phonon lines observed in lightly doped cuprates [36] and nickelates [25,34].

2. The temperature dependencies of the frequency position and integrated intensity for the $\sim 730 \mathrm{~cm}^{-1}$ peak differ from those of the one-phonon peak at $684 \mathrm{~cm}^{-1}$ in undoped $\mathrm{La}_{2} \mathrm{NiO}_{4.0}$ and of one-phonon peaks at lower frequencies in doped nickelates and are similar to the corresponding dependences for the second wide band at $\sim 1120 \mathrm{~cm}^{-1}$.

3. In our RS spectra two sharp lines at 580 and $630 \mathrm{~cm}^{-1}$ are superposed on the broad band (see Fig. 6). Moreover, the Fano lineshape seen for the $580 \mathrm{~cm}^{-1}$ line is clearly seen in Fig. 8. The Fano effect in Raman scattering is observed as a characteristic change in the usually Lorentzian-lineshape of phonon peaks in the spectra - the phonon lineshape becomes asymmetric. It means that a coherent interaction exists between the two scattering sources. We believe that the observed effect is connected with an interaction between the phonon, which shows a Fano effect and the charge carriers excitations [12] (dashed line in Fig. 8) causing the background. However, we do not exclude the possibility of an interaction with the excitation causing the wide band at $\sim 730 \mathrm{~cm}^{-1}$. The probability of the latter assumption is now analyzed theoretically.

In Ref. 35 only one band was attributed to two-magnon scattering in the case where the spin exchange occurs near the diagonal charge domain wall. However, it was examined the case of one domain wall without taking into account the width of 


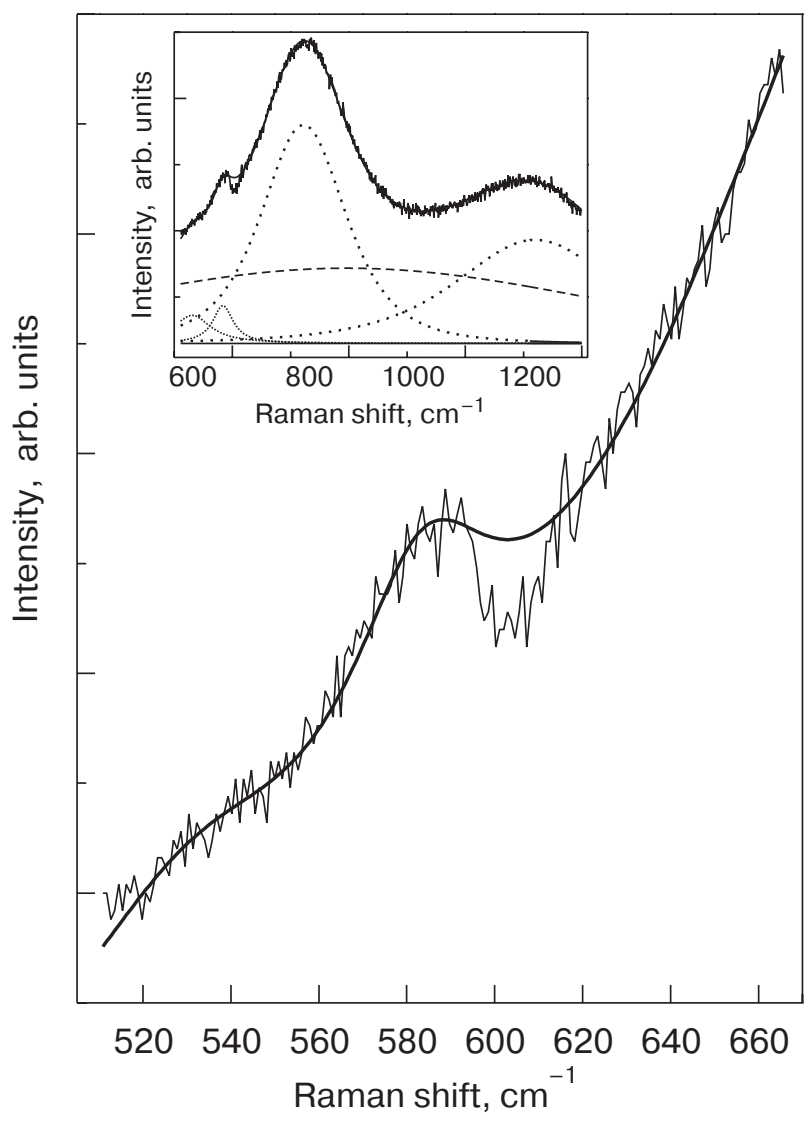

Fig. 8. Fano lineshape of the phonon at $580 \mathrm{~cm}^{-1}$ for $x^{\prime} y^{\prime}$ polarization of $\mathrm{La}_{5} /{ }_{3} \mathrm{Sr}_{1} /{ }_{3} \mathrm{NiO}_{4}$ single crystal at $T=5 \mathrm{~K}$. The solid line is a theoretical fit to the experimental curve using Lorentzian-lineshape of phonon peak. Inset show experimental spectrum in the frequency region of 500-1200 $\mathrm{cm}^{-1}$. Dot lines represent a fit with Lorentzian lineshapes, dashed line is a charge carriers scattering [12].

antiferromagnetic domain that lead to incorrect calculation of nearest neighbors number.

Not any feature was observed in the single-magnon dispersion which would correlate with the lower-energy two-magnon peak in the inelastic neutron scattering measurements of the stripe-ordered nickelate $\mathrm{La}_{1.69} \mathrm{Sr}_{0.31} \mathrm{NiO}_{4}$ also [37].

Next we have tried to analyze the two-magnon scattering theoretically. A simple square plane array of paramagnetic ions was implemented for the calculations. The site-centered model of charge ordering was used, and thus paramagnetic ions inside the domain walls were considered to be frustrated. So, the pattern of spins for approximation of the $\mathrm{La}_{5}{ }_{3} \mathrm{Sr}_{1}{ }_{3} \mathrm{NiO}_{4}$ magnetic structure was similar to those showed in Fig. 1, $a$ or in Ref. 21. Four exchange integrals between nearest and next-nearest neighbors were taken into account. Two of them are found to be identical to $J$ and $J^{\prime}$ labeled exchange integrals introduced in Ref. 21. The exact solution for two-magnon light scat- tering line shape was obtained with the following restrictions: (i) zero temperature or temperature much smaller than $T_{N}$; (ii) Heisenberg character of spin exchange; (iii) small single-ion anisotropy, in comparison with exchange energy. Two-magnon band shape was calculated in the exchange approximation of the Moriya theory using real polarizability tensors connected with the respective exchange integrals.

Before the appearance of Ref. 21 the values of exchange integrals were unknown. We did only estimates using the value for undoped $\mathrm{La}_{2} \mathrm{NiO}_{4}$ for an exchange integral which bonds spins inside a single antiferromagnetic domain. By the way, our previous attempts to approximate both bands failed. The calculated shapes cannot be fitted to the experimental spectra at any value of exchange integrals and respective values of polarizability constants we used.

Recent experimental data of neutron inelastic scattering [21] have just supplied the needed exchange integral values. Using those we have obtained a reasonably good description of the band at $\sim 1110 \mathrm{~cm}^{-1}$ with the following values of the exchange integrals and respective polarizability constants relation: $J=$ $=242 \mathrm{~cm}^{-1}(30 \mathrm{meV}), J_{2}=109 \mathrm{~cm}^{-1}(13.5 \mathrm{meV})$, $P_{2} / P=-0.75$. Our values of exchange integrals are twice higher then the respective values of $J$ and $J^{\prime}$

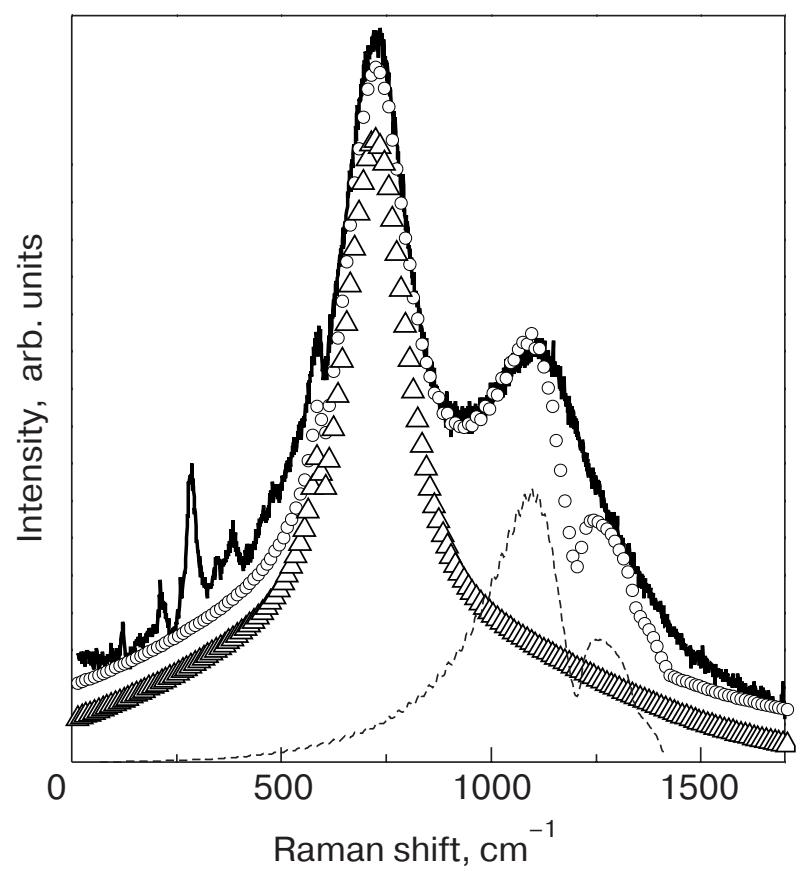

Fig. 9. Theoretical approximation of Raman spectra for $x^{\prime} y^{\prime}$ polarization of $\mathrm{La}_{5}{ }_{3} \mathrm{Sr}_{1 / 3} \mathrm{NiO}_{4}$ single crystal at $T=5 \mathrm{~K}$. Solid line is the experimental spectrum, dashed line is the calculated two-magnon band, triangles present a sum of some reasonable spectral shapes to fit band at $\sim 720 \mathrm{~cm}^{-1}$ and wide background, open circles represent total fitting spectrum. 
from Ref. 21 due to a different kind of summation over the spin pairs in the Hamiltonians. The result of the best fit is shown in Fig. 9. It is clear that narrow decrease of intensity at $\sim 1200 \mathrm{~cm}^{-1}$ in the theoretical two-magnon band (dashed curve in Fig. 9) has interference nature and probably is a result of above-mentioned restrictions connected with the real type of polarizability constants. Because of the absorption at the exciting laser wavelength in the RS experiments in $\mathrm{La}_{5} /{ }_{3} \mathrm{Sr}_{1 / 3} \mathrm{NiO}_{4}$, it is reasonable to use complex type of these constants. Such calculations are now in progress.

These results show evidence for not simple two-magnon nature of the band at $\sim 730 \mathrm{~cm}^{-1}$. Perhaps possible effects connected with the interaction between spin excitations and collective motion of charge domain-walls $[21,38]$ are necessary to be taken into account.

As it was shown in the neutron diffraction experiments [18], the application of a magnetic field in the regime $T>T_{m}$ induces a staggered magnetic order of period $3 a$ due to the net magnetic moment of the high-temperature bond-centered stripes, together with the odd number of $\mathrm{Ni}$ spins across an antiferromagnetic domain. To test the effect of a magnetic field on stripe ordering, we performed a RS experiments on a piece of the same $\mathrm{La}_{1.775} \mathrm{Sr}_{0.225} \mathrm{NiO}_{4}$ crystal that we have been characterized in detail elsewhere $[12,15,26]$. In this sample the hole density per Ni site along a stripe is significantly less than 1 (electron filling fraction $\delta>0$ ) in contrast to the $x=1 / 3$ sample, where the density is exactly 1 . The sample was cooled from $295 \mathrm{~K}$ to $5 \mathrm{~K}$ in the magnetic field of $0.5 \mathrm{~T}$ and after that the field was switched off RS experiments in quasi-backscattering geometry were performed.

Representative scans are shown in Fig. 10. As it is seen from Fig. 10, $a$, freezing in a magnetic field does not affect the spectra measured in $x^{\prime} x^{\prime}$ scattering geometry. A surprising result was obtained in $x^{\prime} y^{\prime}$ scattering geometry (Fig. 10,b). The second band at $\sim 1130 \mathrm{~cm}^{-1}$ disappears nearly completely (some excess Raman signal above the background is still visible) after freezing the sample in the magnetic field. It is clear, that applied magnetic field leads to a stripe structure reorganization. However, to understand fully the observed effects, further experimental and theoretical efforts are needed. It is supposed, for example, to examine the situations of:

i) Low-temperature bond-centered stripes with a period of $2 / 3 a$. In this state the domains are 3 spins wide and an uncompensated moment appear. The adjacent across the domain wall spins are ferromagnetically aligned.

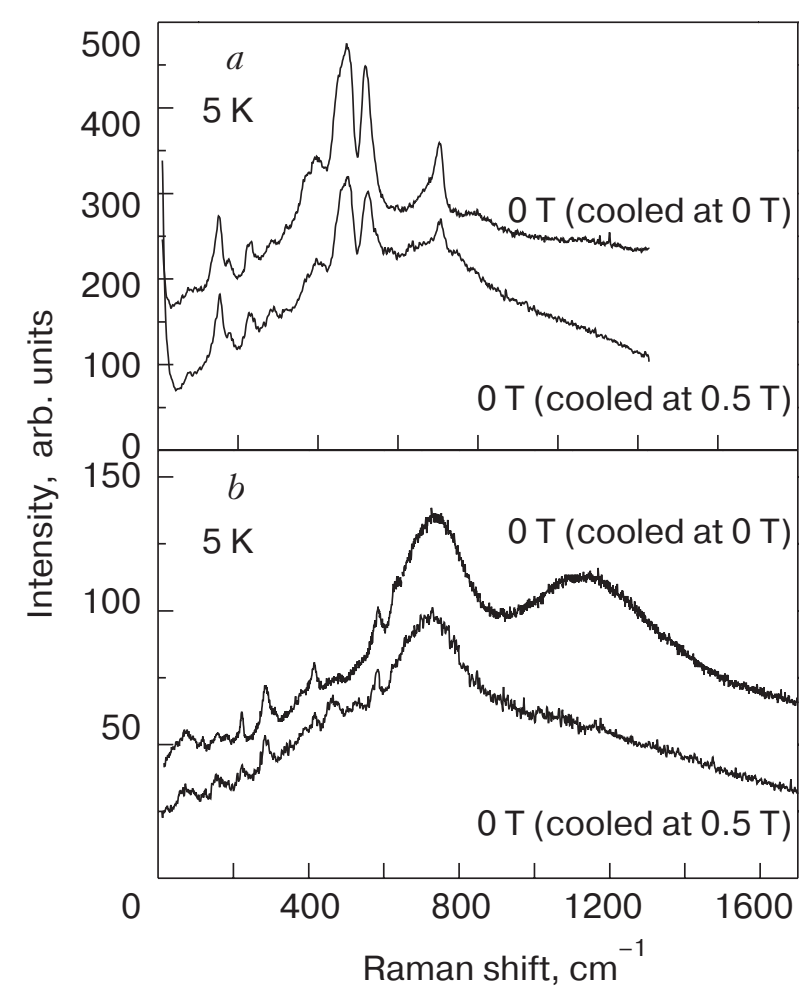

Fig. 10. Raman spectra in $x^{\prime} x^{\prime}(a)$ and $x^{\prime} y^{\prime}(b)$ polarization configuration of the $\mathrm{La}_{1.775} \mathrm{Sr}_{0.225} \mathrm{NiO}_{4}$ crystal cooled in zero and $0.5 \mathrm{~T}$ magnetic field.

ii) In-phase domain walls. As it was experimentally established, neighboring antiferromagnetic domains, separated by a charge stripe, have an antiphase relationship. But in contrast with common folklore, it was recently shown theoretically [39] that the hole rich stripes are not necessary antiphase domain walls of antiferromagnetic spin domains and a phase transition from antiphase to in-phase domain-wall configuration has to occur as a function of increased electron filling fraction of the domain wall. Moreover, «empty» domain walls are always antiphase.

\section{Concluding remarks}

Although the basic nature of the charge and associated spin order in cuprates and nickelates has now been fairly well been established, many questions concerning this order remain to be answered. One of our goals here was to elucidate some problems that are under discussion at present. Unquestionable, Raman scattering will continue to be an essential tool as we try to improve our understanding of stripe ordering and other complex correlation effects.

This work was supported by NATO Collaborative Linkage Grant PST.CLG.977766, INTAS Grant 96-0410, and Ukrainian Grant № 3-026. 
1. S.M. Hayden, G.H. Lander, J. Zaretsky, P.B. Brown, C. Stassis, P. Metcalf, and J.M. Honig, Phys. Rev. Lett. 67, 1061 (1992).

2. K. Yamada, T. Omata, K. Nakajima, Y. Endoh, and S. Hosoya, Physica C221, 355 (1994).

3. C.H. Chen, S.-W. Cheong, and A.S. Cooper, Phys. Rev. Lett. 71, 2461 (1993).

4. J.M. Tranquada, D.J. Buttrey, V. Sachan, and J.E. Lorenzo, Phys. Rev. Lett. 73, 1003 (1994).

5. J.M. Tranquada, J.E. Lorenzo, D.J. Buttrey, and V. Sachan, Phys. Rev. B52, 3581 (1995).

6. J.M. Tranquada, B.J. Sternlieb, J.D. Axe, Y. Nakamura, and S. Uchida, Nature 375, 561 (1995); J.M. Tranquada, J.D. Axe, N. Ichikawa, Y. Nakamura, S. Uchida, and B. Nachumu, Phys. Rev. B54, 7489 (1996).

7. J.D. Axe, H. Moudden, D. Hohlwein, K.M. Mohanty, A.R. Moodenbaugh, and Youwen Xu, Phys. Rev. Lett. 62, 2751 (1989).

8. P. Dai, H.A. Mook, and F. Dogan, Phys. Rev. Lett. 80, 1738 (1998); H.A. Mook, P. Dai, S.M. Haugen, G. Aeppli, T.G. Perring, and F. Dogan, Nature 395, 580 (1998)

9. J. Gopalakrishnan, G. Colsmann, and B. Reuter, J. Solid State Chem. 22, 145 (1977).

10. R.J. Cava, B. Batlogg, T.T. Palstra, J.J. Krajewsky, W.F. Peck, Jr., A.P. Ramirez, and L.W. Rupp, Phys. Rev. B43, 1229 (1991).

11. T. Strangfeld, K. Westerhold, and H. Bach, Physica C183, 1 (1993).

12. V.P. Gnezdilov, Yu.G. Pashkevich, A.V. Yeremenko, P. Lemmens, G. Guntherodt, J.M. Tranquada, D.J. Buttrey, and K. Nakajima, Fiz. Nizk. Temp. 28, 716 (2002) [Low Temp. Phys. 28, 510 (2002)].

13. Takuya Noda, Hiroshi Eisaki, and Shin-Ichi Uchida, Science 286, 265 (1999).

14. O. Zachar, S.A. Kivelson, and V.J. Emery, Phys. Rev. B57, 1422 (1998).

15. J.M. Tranquada, D.J. Buttrey, and V. Sachan, Phys. Rev. B54, 12318 (1996).

16. S.-W. Cheong, H.Y. Hwang, C.H. Chen, B. Batlogg, W. Rupp, Jr., and S.A. Carter, Phys. Rev. B49, 7088 (1994).

17. V. Sachan, N. Hamada, K. Terakura, and T. Asada, Phys. Rev. B53, 12742 (1995).

18. J.M. Tranquada, P. Wochner, A.R. Moodenbaugh, and D.J. Buttrey, Phys. Rev. B55, R6113 (1997).
19. H. Yoshizawa, T. Kakeshita, R. Kajimoto, T. Tanabe, T. Katsufuji, and Y. Tokura, Phys. Rev. B61, R854 (2000)

20. S.-H. Lee, S.-W. Cheong, K. Yamada, and C.F. Majkrzak, Phys. Rev. B63, 060405 (2001).

21. A.T. Boothroyd, D. Prabhakaran, P.G. Freeman, S.J.S. Lister, M. Enderle, A. Heiss, and J. Kulda, Phys. Rev. B67, 100407(R) (2003).

22. Jianqi Li, Yimei Zhu, J.M. Tranquada, K. Yamada, and D.J. Buttrey, Phys. Rev. B67, 012404 (2003).

23. G. Blumberg, M.V. Klein, and S.W. Cheong, Phys. Rev. Lett. 80, 564 (1998)

24. K. Yamamoto, T. Katsufuji, T. Tanabe, and Y. Tokura, Phys. Rev. Lett. 80, 1493 (1998).

25. S. Sugai, N. Kitamori, S. Hosoya, and K. Yamada, J. Phys. Soc. Jpn. 67, 2992 (1998)

26. Yu.G. Pashkevich, V.A. Blinkin, V.P. Gnezdilov, V.V. Tsapenko, V.V. Eremenko, P. Lemmens, M. Fisher, M. Grove, G. Guntherodt, L. Degiorgi, P. Wachter, J.M. Tranquada, and D.J. Buttrey, Phys. Rev. Lett. 84, 3919 (2000).

27. D.J. Buttrey, H.R. Harrison, J.M. Honig, and R.R. Schartman, J. Solid State Chem. 54, 407 (1984).

28. F.E. Bates and J.E. Eldridge, Solid State Commun. 72, 187 (1989)

29. G. Burns, F.H. Dacol, D.E. Rice, D.J. Buttrey, and M.K. Crowford, Phys. Rev. B42, 10777 (1990).

30. de Anders et al., J. Phys.: Condens. Matter 3, 3813 (1991).

31. M. Udagawa, T. Yamagichi, Y. Nagaoka, N. Ogita, M. Kato, Y. Maeno, T. Fujita, and K. Ohbayashi, Phys. Rev. B47, 11391 (1993).

32. V.I. Anisimov, M.A. Kotorin, J. Zaanen, and O.K. Anderesen, Phys. Rev. Lett. 68, 345 (1992).

33. P. Wochner, J.M. Tranquada, D.J. Buttrey, and V. Sachan, Phys. Rev. B57, 1066 (1998).

34. S. Sugai, M. Sato, T. Kobayashi, J. Akimitsu, T. Ito, H. Takagi, S. Ushida, S. Hosoya, T. Kajitani, and T. Fukuda, Phys. Rev. B42, 1045 (1990); S. Sugai, S. Hosoya, T. Kajitani, T. Fukuda, and S. Onodera, Physica C185-189, 895 (1991).

35. S. Sugai and N. Haumizu, Stripes AIP Conf. Proc. 554, 84 (2001)

36. S. Sugai, Phys. Rev. B39, 4306 (1989).

37. P. Bourges, Y. Sidis, M. Braden, K. Nakajima, and J.M. Tranquada, Phys. Rev. Lett. 90, 147202 (2003).

38. J. Zaanen, M.L. Horbach, and W. van Saarloos, Phys. Rev. B53, 8671 (1996).

39. O. Zachar, Phys. Rev. B65, 174411 (2002). 